\title{
Review Reports on User Authentication Methods in Cyber Security
}

\author{
ARTI VAISH $^{1 *}$, ANAND SHARMA ${ }^{2}$, ANSHU SHARMA ${ }^{3}$ \\ ${ }^{1,2}$ School of Engineering and Technology, ${ }^{3}$ Organizational Behavior and Human Resource \\ Management \\ ${ }^{1,2}$ Ansal University, ${ }^{3}$ O.P. Jindal University \\ ${ }^{1,2}$ Ansal University, Sector -55, Golf Course University, Gurgaon and ${ }^{3}$ Narela Road, Sonepat \\ $1,2,3$ INDIA
}

\begin{abstract}
The Internet has merged itself as an extremely ground- breaking stage that has changed the correspondence and business exchanges. Presently, the quantity of clients exploring the Internet is more than 2.4 billion. This enormous group of spectators requests online business, learning sharing, informal organizations and so on, which became exponentially in the course of recent years. Accordingly, it prompts the requirement for security and improved protection. As of late, misrepresentation over the Internet comprises one of the fundamental disadvantages for the across the board of the utilization of business applications. Along these lines, the three imperative security issues occur each day in our universe of straightforward design, even more decisively: recognizable proof, confirmation and approval. Distinguishing proof is a procedure that empowers acknowledgment of a substance, which might be either, a human, a machine, or another advantage, for example, a product program. In security frameworks, validation and approval are two reciprocal systems for figuring out who can get to the data assets over a system. Numerous arrangements have been proposed in the writing, from a straightforward secret phrase to late advancements dependent on RFID (Radio Frequency Identification) or biometrics. This paper gives an outline on existing verification techniques, and its upsides and downsides when planning online assistance.
\end{abstract}

Keywords: Information technology, user authentication, online services, cryptography, biometrics

Received: March 17, 2020 . Revised: September 12, 2020. Accepted: October 1, 2020. Published: October 6, 2020.

\section{Introduction}

As far back as two decades, PC frameworks have created at a risky rate. In a wide extent of conditions, such frameworks have transformed into a significant contraption. Affiliations are building frameworks with greater scales than at some other time, and the system with the overall Internet has ended up being fundamental. Nearby this example has come an impact on the usage of PC composes as a strategy for illicit access to PC structures. The web is known as a historic stage that changes the way wherein we grant and perform business trades in current advancement [1]. It has now reached each piece of our lives nearby ascending of additional cutting-edge security risks, arranged to leave towards the experience of annihilations. As shown by the Internet World Stats, as of June 30, 2012, over 2.4 billion customers are using the Internet, and accordingly the numbers no vulnerability will keep growing. Thusly, the presence of information assurances has changed our lives particularly with the information that is available, whereby data can without quite a bit of a stretch be gotten to and controlled [2]. Transmitted information level is twisting up logically huge especially as correspondences that used to simply be finished disengaged, for instance, bank and business exchanges are by and by being done online as
Internet banking and electronic business exchanges, and damages on account of such attacks will be increasingly conspicuous. As extending proportions of individual information are surfacing on the Web, it is essential to remain cautious about the threats incorporating the straightforwardness wherein our private nuances can be gotten to. Relational collaboration and online profiles add to this: giving potential gatecrashers a lot of delicate information [3-4]. Insafe reports that more than a fourth of children in Europe have web frameworks organization profiles, which can be revealed, and within excess of 900 million people on Facebook alone, the hazard is expansive.

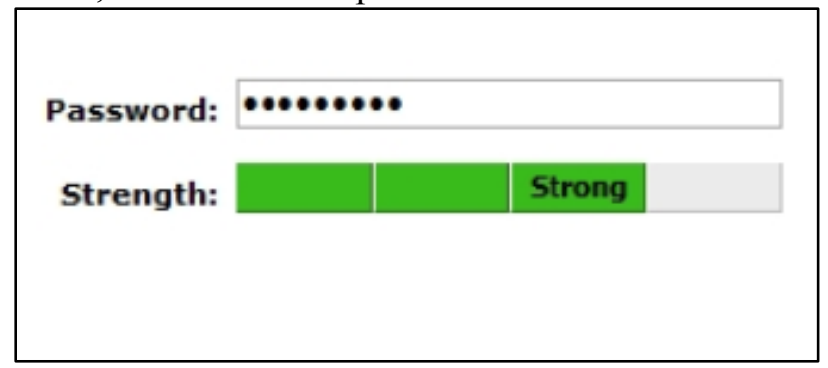

Fig. 1 Password showing strong strength

\section{Concepts Authentication}

Before introducing the diverse existing strategies, we give a few definitions and ideas. The confirmation procedure suggests various substances: 
1. The inquirer is the substance that confirms to the framework, so as to utilize the administrations. It could be an individual or an Information System (IS);

2. The screen is the substance that gives a confirmation administration. It affirms the character of an inquirer (or reject it if there should be an occurrence of an off-base confirmation) and checks on the off chance that it can concede him/her the utilization of the necessary help;

3. The Information System (IS) gives administrations, for example, an entrance to a PC account, an application, an entryway opening or a system printer, and will give the inquirer a chance to utilize its administrations if the screen accurately confirmed it (with a given required degree of trust).

\section{The Authentication Concept:}

'Recognizing confirmation', 'approval' and 'endorsement' are three interrelated thoughts, which structure the focal point of a security system. The ID is the correspondence of a character to an IS. Before check, the candidate ordinarily gives the IS a character regardless (for example, a login or an email address), and the screen expresses the character by approval (for example, using a mystery expression). Approval is a proof given by an inquirer to attest a screen that he/she genuinely looks at to the character he/she gave [5]. The screen by then confirms the IS of the character of the customer. Finally, endorsement is the yielded advantages given to the customer. Do approval systems offer reactions to the two request: (I) who is the customer? Also, (ii) is essentially the customer really who he/she addresses himself/herself to be? In this way, affirmation addresses one of the most promising ways concerning trust and security update for business applications. It is like manner connotes a property of ensuring the character of the as of late referenced components. Moreover, endorsement is a strategy of giving individuals a passage to the structure articles subject to their character. Endorsement structures give the reactions to the three request: (I) is customer $\mathrm{U}$ affirmed to get to resource R? (ii) is customer $\mathrm{U}$ endorsed to perform movement $\mathrm{O}$ ?; and (iii) is customer $\mathrm{U}$ endorsed to perform action $\mathrm{O}$ on resource $\mathrm{R}$ ? There is as often as possible confusion between 'ID', 'check' and 'endorsement'. These words/terms do not have a comparable criticalness using any and all means. All of these thoughts requires an enrolment step. Enrolment is the 'enlistment' of another customer, including the surge of tokens and confirmations. Enrolment is a noteworthy concern and should in like manner be carefully dealt with. In the rest of this paper, we will consider the IS has recently taken on the inquirer.

A channel is help of correspondence between the inquirer and the screen. It can either be considered as grouped, legitimate, secure or as insecure. A mystery channel is impenetrable to catch endeavor; a genuine channel is impenetrable to modifying; an ensured channel is impenetrable to both, and a temperamental channel is none [6]. The approval objective is to express a character, be that as it may, the degree of affirmation methods is amazingly gigantic and it can vary from different perspectives. Coming up next is a summary of a bit of the essential approval procedures:

An ID (IDentification)/password: to open a session on a computer or to authenticate on Internet;

1. A PIN (Personal Identification Number) code: to unlock a smartcard;

2. An RFID card: for accessing a building;

3. A fingerprint: to unlock a gateway;

4. A facial recognition system with webcam;

5. A USB token;

6. A one-time password token

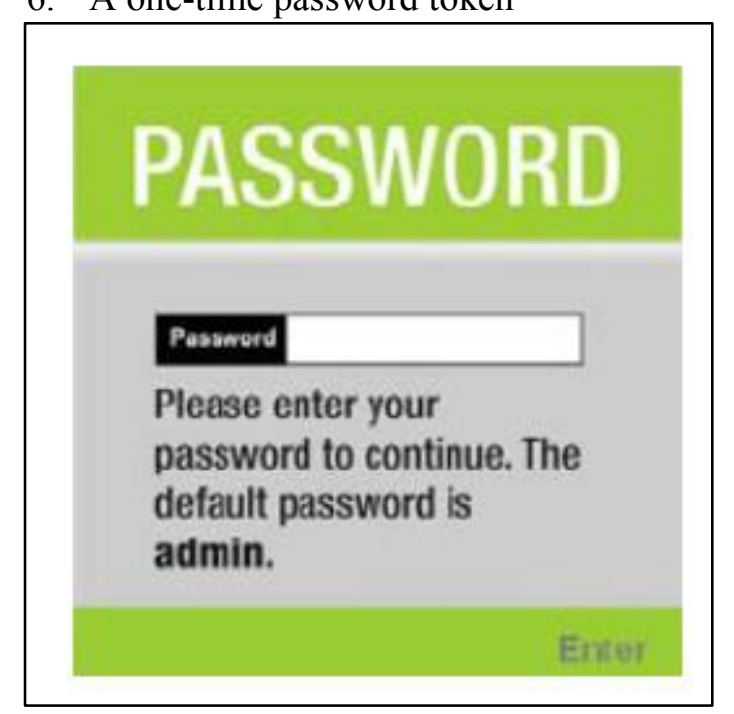

Fig. 2 An ID (IDentification)/password

\section{Basic Steps for Authentication:}

The common basic phases for authentication are: 1. Introductory advance: the individual is unauthenticated.

2. Association step: the individual needs to the is that the utilization of a work that needs confirmation. The IS requests that the screen bear witness to the person. 3. Attested step: the individual is attested and a session is opened. The IS provides the user the specified functions.

4. Disconnection step: the user disconnects or is disconnected from the monitor and also the state returns to the initial step. 
A channel is the guide of correspondence between the inquirer and the screen. It can be considered as private, true blue, either secure or as dubious [7]. A mystery channel is impenetrable to an IS may require different degrees of approval, for example, a level for the directors and a level for the customers. In such a structure, the level of affirmation is graduated on a scale: level 0 for an unauthenticated customer with minimal rights in the system; level $\mathrm{N}$ for the director with full rights; and one or different levels among 0 and $\mathrm{N}$. Here, the arrangement is that a confirmation could be required to change to a progressively raised degree of trust in the inquirer by the IS. They gave security of an affirmation system that endless supply of utilization and appropriateness. If the usability is awful, the customers will rapidly find ways to deal with evade the approval adventures for accommodating use. This will lead unavoidably to a failure of the system, so it should be considered as an intellectual [8].

\section{Authentication Factors and Strengths}

Utilizing more than one factor to verify a client is at times related as solid validation, yet the quality of the confirmation is progressively identified with the quality of underneath verification techniques, to be increasingly exact: "Two-Factor Authentication" (TF-A). For instance, a TF-A suggests a pilot treat (what you claim) and the capacity to give the mother's last name by birth (what you know) is a TF$\mathrm{A}$, not a strong validation, as every verification way will be essentially undermined. Another elective factor could be:

- A spatiotemporal approval: the customer can be offered access to his/her working environment exactly at some predefined times and territories;

- A web of trust: reputation could be a factor for checking once in a while.

- A Turing test (Turing, 1950) could be considered as approval as a human instead of a PC (a CAPTCHA is an instance of a Turing test).

\section{Cryptographic Challenge-Response Based Authentication}

PAP (Password Authentication Protocol) is an essential show for check over a framework, which sends clear passwords and identifiers over the framework. As such, CHAP (Challenge Handshake Authentication Protocol) is an improvement of PAP, yet in any case, it requires transmitting a hashed mystery word. The guideline thought of a test response based affirmation is that the inquirer shows he/she knows the puzzle without sending it clear over the channel. Along these lines, CHAP is a test based affirmation show, be that as it may, the transmission of a hashed mystery state is so far an issue on account of savage power and vocabulary ambushes [9]. Likewise, hashed passwords still contain a lot of information about the baffling mystery key. The principal response to handle that issue is the usage of cryptography, either symmetric or hilter kilter in order to execute a test response confirmation. When in doubt, a test response confirmation structure is a system that issues a "challenge" on the client interest for instance challenge in this particular circumstance: question of ID, and checks it in the "response" of the ensured character for instance response in this particular condition: give an exhibit of recognizing verification. In the symmetric case, the screen sends a test to the solicitor. The test can be a tremendous entire number, an assortment of numbers... All things considered, the inquirer by then enciphers the test with the regular key and sends the result back to the screen, which differentiates the result and the one it has also decided. In the uneven case, the inquirer has a private key identified with an affirmation that contains the relative open key. The candidate gives the screen his/her confirmation (and his/her open key). The screen sends a subjective number as a test, and the applicant uses its private key to sign the test (or to encipher it) and sends the result back to the screen. The screen by then affirms the imprint with the open key (or unwind the response) to check the test was checked [10].

\section{Radio Frequency Identification:}

The first of RFID names were used during the 1950s for a military explanation, anyway, it was obliged to a conspicuous verification reason. Around that point, their usage was obliged to the transmission of a successive number remotely over radio waves. In the past couple of years, RFID marks have been a tremendous achievement in the industry, and their use wound up typical. Application extent of RFID marks is gigantic: thing ID for stock data, store organizes the officials, phones with RFID limits, check plastic cards... It is said that we depend on the Internet for things, and in this way, RFID marks will help to progress toward thusly by adding correspondence abilities to ordinary things. The closeness of RFID names in the normal everyday presence has as of late begun. For example, an accuse card of an inalienable RFID structure could be charged by a remote POS (Point of Sale), paying little respect to whether the card is as yet dealt with its owner [11]. The old-style protection from those attacks is a PIN code engraved on the card, which is 
relied upon to open the chip. So the assailant ought to have the card close by to examine the code. Such security is used for RFID recognizable pieces of proof (biometric international IDs).

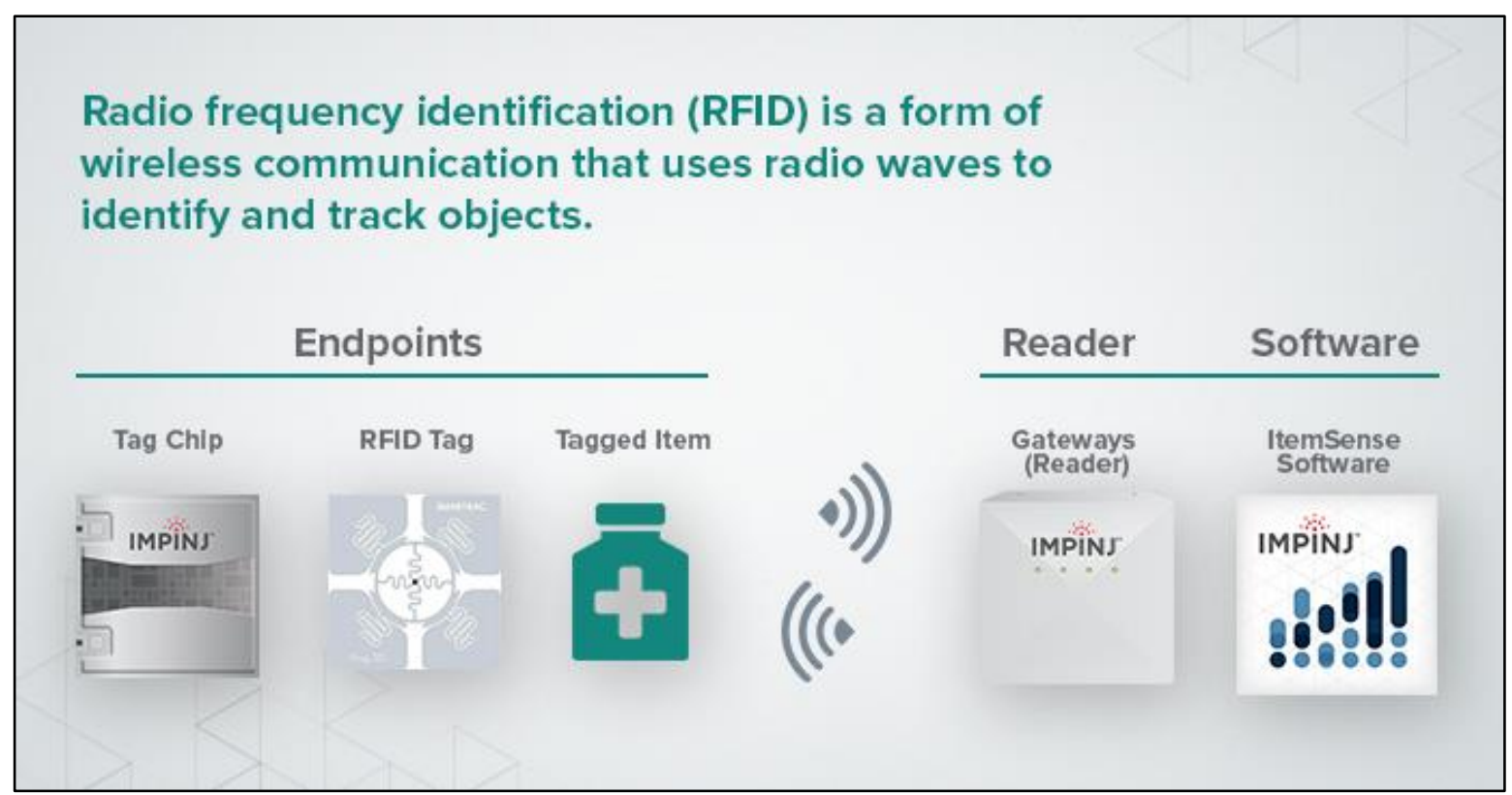

Figure 3: Working of an RFID

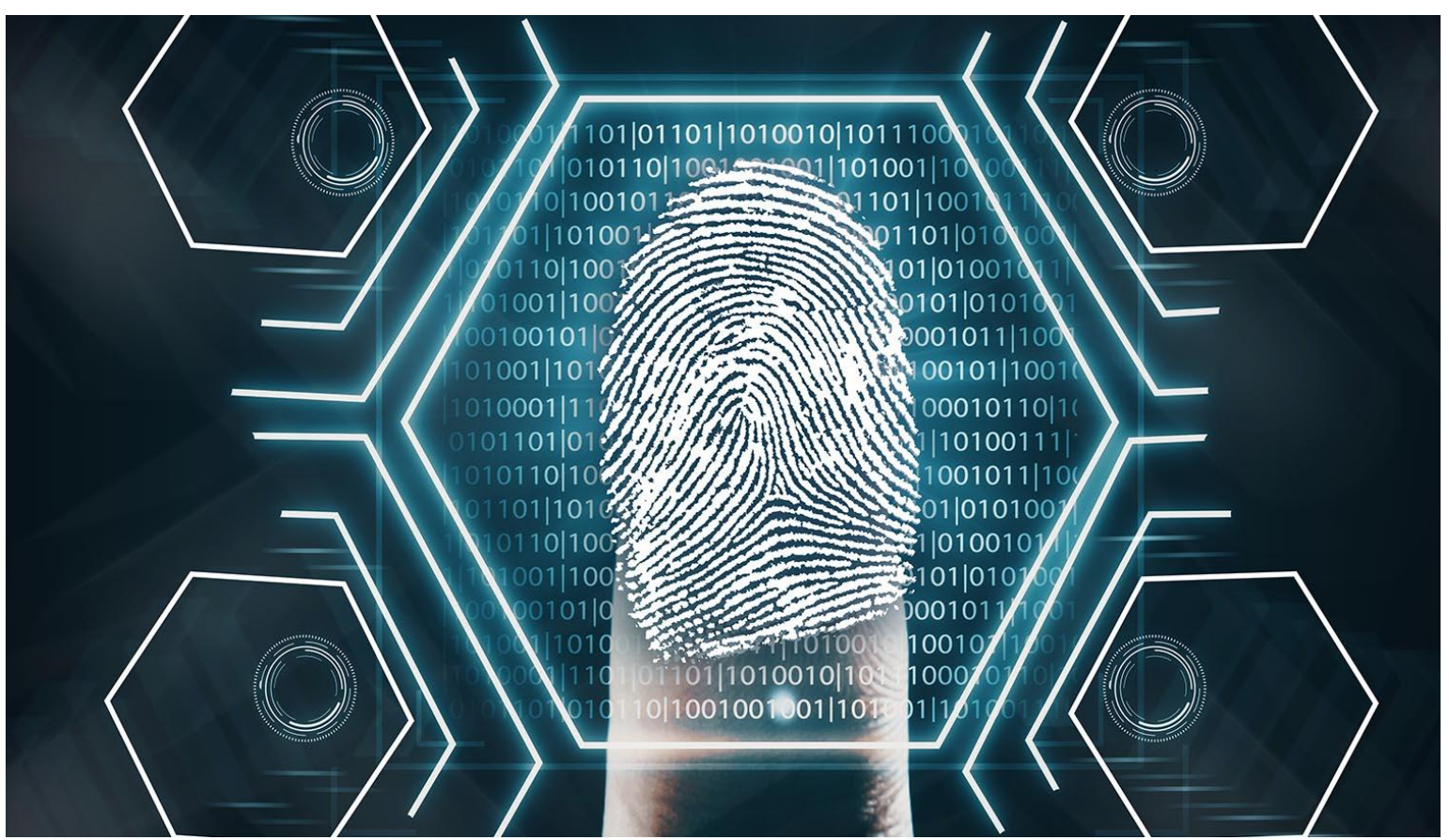

Figure 4: Basics of biometrics

\section{Biometrics:}

In software engineering, specifically, biometrics is utilized as a type of personality get to the executives and access control.

Biometrics has been around since around 29,000 $\mathrm{BC}$ when stone-age men would sign their drawings with impressions. In $500 \mathrm{BC}$, Babylonian business trades were set apart in mud tablets with fingerprints. The soonest arranging of fingerprints returns to 1891 when Juan Vucetich started a gathering of fingerprints of punks in Argentina. Regardless, it is said that the verifiable scenery of biometrics frameworks started in China in the fourteenth century. It was a sort of fingerprinting as uncovered by the Portuguese understudy of history Joao de Barros. The Chinese merchants were venturing children's palm and impacts on paper with ink to perceive babies. Biometric affirmation is, for the most part, inspected in programming designing. The usage of biometric frameworks, for instance, face, fingerprints, iris, and 
ears is a response for getting a sheltered individual check procedure. Biometrics uses the approval factors, which are techniques subject to something that qualifies the customer and something that he/she can do. The basic piece of a room of these confirmation methodologies is that there exists a strong association between the individual (customer) and its authenticator (biometric data). Moreover, it is difficult to copy the biometric characteristics of an individual diverged from most by far of other approval strategies. In any case, there is a disservice in biometric approval, which is the helplessness of its check result, for example, in fingerprints affirmation; there could be a possible bumble due to dreadful arranging of the finger. Biometric approval can be dense in two phases to be explicit enrolment and affirmation. The period of the enrolment is the spot the customer gives his/her biometric data. The biometric data will be gotten and from that point onward, the features will be isolated and sent away into the database. During the approval strategy, the put away features will be differentiated and the ones right currently presented for passage. In case it matches, by then, a passageway will be permitted. For example, in keystroke components, during the enrolment mastermind, the customers are requested to give their way from forming for instance by creating given a mystery key or a passphrase on the support between 5 to various occasions. Since keystroke components are lead biometrics, along these lines, it must be done all in all, for instance, a couple of amounts of times in light of the fact that each time, the way wherein the customers type a mystery expression/passphrase, their creating mindset may differentiate fairly.

\section{Conclusion}

The principal future pattern is obviously to expand the security of data frameworks through a safe validation of the person. Implying that we need to be as certain as conceivable that the petitioner isn't an impostor. The diverse validation arrangements that could be conveyed must be straightforward for a customer. Validation is a piece of the human machine interface for any product applications. The usability is significant for the sensible access control step yet additionally for the enrolment one. As, some data frameworks require a solid secret word that will incorporate a blend of capitalized and lowercase letters, numbers, and images. In the event that the secret word isn't sufficient the individual is approached to pick another. This is commonly badly arranged for the client to characterize such a secret key and to recollect it (Park et al., 2004). Modern and analysts by and large characterize effective and secure confirmation arrangements as a specialized perspective. A decent pragmatic arrangement will think of some as ease of use perspectives during the improvement step. For some ergonomic or social reasons, a confirmation technique would not be acknowledged by clients. As an outline, even secret word composing can be hesitant for certain clients. Human memory is in strife with most secret phrase strategies. The creators note the ease of use issues with secret phrase validation, for example, the quantity of passwords a client needs to recall, exacting secret key arrangements, differing frameworks, and memory requests. Clients once in a while totally overlook a secret key. Numerous human factor investigations of information passage techniques have been acknowledged before.

Funding: This study has not funded by any authority. Conflict of Interest: The authors declare that they have no conflict of interest.

\section{References:}

[1] Syed Idrus, S.Z., E. Cherrier, C. Rosenberger and P. Bours, A preliminary study of a new soft biometric: Finger recognition for keystroke dynamics, In 9th Summer School for Advanced Studies on Biometrics for Secure Authentication: Understanding Man Machine Interactions in Forensics and Security Applications, 2012b.

[2] Chabaud, F. and O. Grumelard, Authentication, a model of human machine authentication, In workshop European Network and Information Security Agency (ENISA), 2006.

[3] Menezes, A., P. Van Oorschot and S. Vanstone, 1996. Identification and Entity Authentication, chapter 10: Manual Guide of Applied Cryptography.

[4] Syed Idrus, S.Z., Database encryption for a web-based claims system. Master's thesis, School of Computer and Communication Engineering, Universiti Malaysia Perlis, Perlis, Malaysia, 2008.

[5] Wikipedia. Biometrics, 2011a. URL http://en.wikipedia.org/wiki/Biometrics.

Wikipedia. Biometric passport, 2011b. URL http://en.wikipedia.org/wiki/Biometrics. J. D. Woodward. Biometrics: Privacys foe or privacys friend? Journal of IEEE, 85(9): 14801492, 1997. J. Yang, editor. Biometrics. InTech, 2011. Yang, J. and L. Nanni, 2011. editors. State of the art in Biometrics. InTech, 2011.

[6] Sridhar, S., and S. Smys. "Intelligent security framework for iot devices cryptography based end-to-end security architecture." In 2017 
International Conference on Inventive Systems and Control (ICISC), pp. 1-5. IEEE, 2017

[7] Sridhar, S., and S. Smys. "A hybrid multilevel authentication scheme for private cloud environment." In 2016 10th International Conference on Intelligent Systems and Control (ISCO), 2016, pp. 1-5.

[8] Goyal, Vipul, et al., The N/R one time password system, International Conference on Information Technology: Coding and Computing (ITCC'05)-Volume II. Vol. 1. IEEE, 2005, pp. 733-738.
[9] Kotzanikolaou, P. and C. Douligeris, 2007. Network Security Current Status and Future Directions, chapter Computer Network Security: Basic Background and Current Issues.

[10] Newham, E., 1995. The biometric report, URL http://www.sjb.com.

[11] Uludag, U., S. Pankanti, S. Prabhakar and A.K. Jain, 2004. Biometric cryptosystems: issues and challenges. In Proceedings of the IEEE 92: 948960

Creative Commons Attribution License 4.0 (Attribution 4.0 International, CC BY 4.0)

This article is published under the terms of the Creative Commons Attribution License 4.0

https://creativecommons.org/licenses/by/4.0/deed.en_US 